Critical phenomena of the disorder driven localization-delocalization transition

by

\author{
Marc Rühländer
}

A dissertation submitted to the graduate faculty

in partial fulfillment of the requirements for the degree of

DOCTOR OF PHILOSOPHY

\author{
Major: Condensed Matter Physics \\ Program of Study Committee: \\ Costas M. Soukoulis, Major Professor \\ Bruce N. Harmon \\ Marshall Luban \\ Constantine Stassis \\ Stephen Willson
}

Iowa State University

Ames, Iowa

2001

Copyright (C) Marc Rühländer, 2001. All rights reserved. 


\section{Graduate College \\ Iowa State University}

This is to certify that the Doctoral dissertation of

Marc Rühländer

has met the dissertation requirements of Iowa State University

Major Professor

For the Major Program 
I dedicate this dissertation to my parents who have supported me throughout this time, even though an ocean separated us and I was not able to visit them as much as I would have liked to. 


\section{TABLE OF CONTENTS}

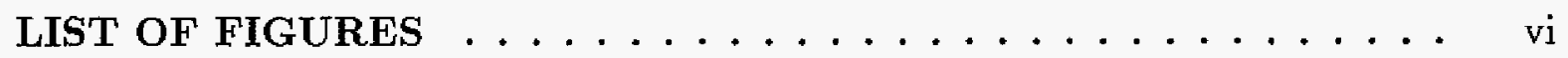

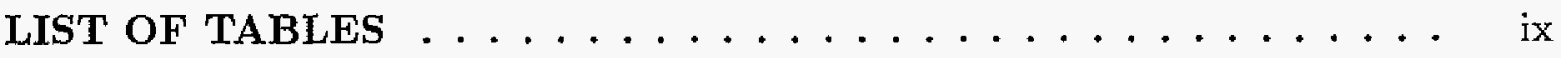

CHAPTER 1. GENERAL INTRODUCTION . . . . . . . . . 1

1.1 Overview of transport in disordered media . . . . . . . . . . . . 1

1.2 Model and methods . . . . . . . . . . . . . . . . 5

1.3 Dissertation Organization . . . . . . . . . . . . . 9

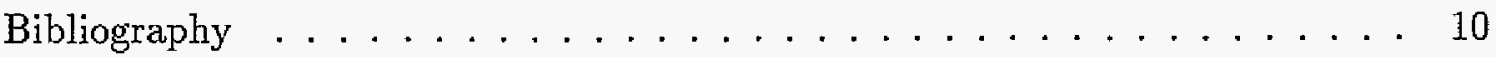

CHAPTER 2. METAL-INSULATOR TRANSITIONS IN ANISOTROPIC TWO-DIMENSIONAL SYSTEMS . . . . . . . . . . . . 13

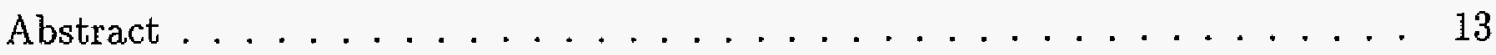

2.1 Introduction . . . . . . . . . . . . . . . . 14

2.2 Model and Methods . . . . . . . . . . . . . . . 15

2.3 Results . . . . . . . . . . . . . . . . . . . 17

2.4 Conclusions . . . . . . . . . . . . . . . . . . . . . 25

Acknowledgements . . . . . . . . . . . . . . . . . 25

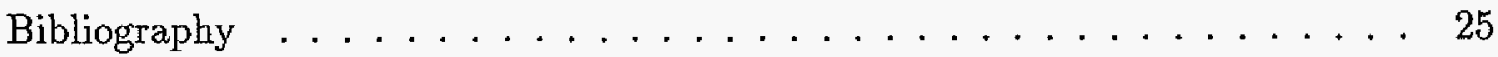


CHAPTER 3. PROBABILITY DISTRIBUTION OF THE CONDUCTANCE IN ANISOTROPIC SYSTEMS . . . . . . . . . . 28

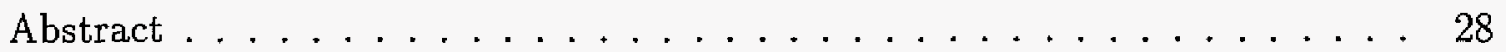

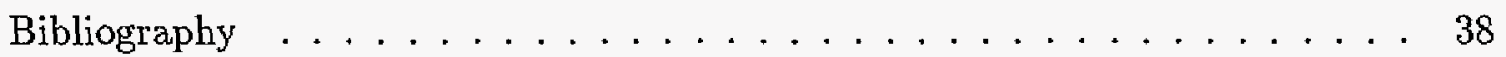

CHAPTER 4. SYMMETRY, DIMENSION AND THE DISTRIBUTION OF THE CONDUCTANCE AT THE MOBILITY EDGE . * 41

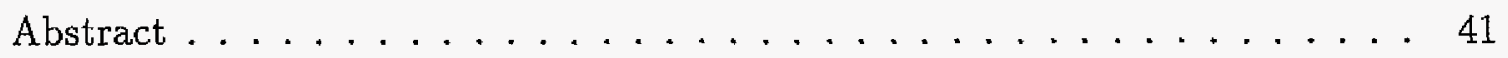

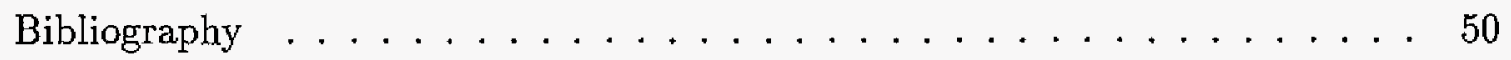

CHAPTER 5. CONDUCTANCE FLUCTUATIONS AND BOUND-

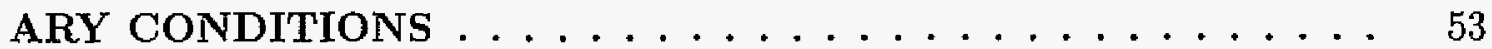

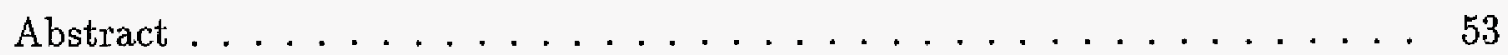

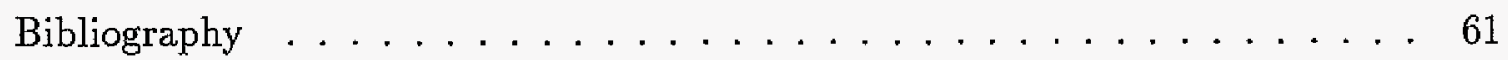

CHAPTER 6. GENERAL CONCLUSIONS . . . . . . . . . . . 62

APPENDIX A. ENERGY LEVEL STATISTICS IN ANISOTROPIC

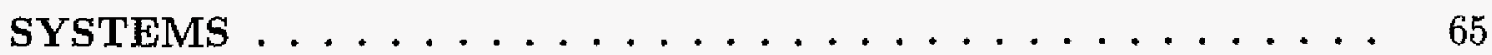

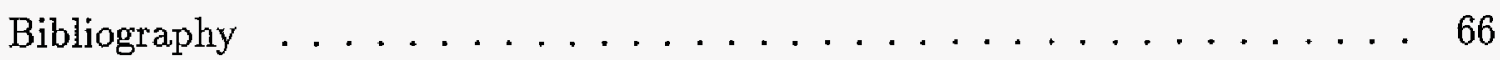

APPENDIX B. MULTIFRACTAL ANALYSIS . . . . . . . . . 70

B.1 Thermodynamics ....................... 70

B.2 Multifractals . . . . . . . . . . . . . . 71

B.3 The inverse participation number ............. 73

B.4 Numerical results . . . . . . . . . . . . . . . . 74 
Bibliography $\ldots \ldots \ldots \ldots \ldots \ldots \ldots \ldots \ldots \ldots \ldots$

APPENDIX C. CONDUCTANCE CALCULATIONS . . . . . . . . 79

Bibliography $\ldots \ldots \ldots \ldots \ldots \ldots \ldots \ldots \ldots \ldots \ldots \ldots$ 


\section{LIST OF FIGURES}

Figure 2.1 Critical values of anisotropic scaling functions . . . . . . . 18

Figure 2.2 Critical value of isotropic scaling function . . . . . . . . . . . 19

Figure 2.3 Anisotropic scaling functions . . . . . . . . . . . . . . 20

Figure 2.4 Rescaled scaling functions . . . . . . . . . . . . . . 21

Figure 2.5 Critical behavior of localization lengths . . . . . . . . . 22

Figure 2.6 Gritical energies are off the band center . . . . . . . . . . . . 24

Figure 3.1 Critical conductance distribution of anisotropic system with magnetic field . . . . . . . . . . . . . . . 35

Figure 3.2 Critical conductance distribution of anisotropic system with very

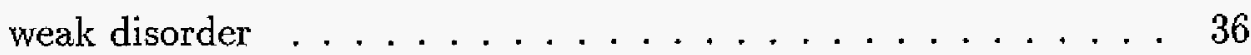

Figure 3.3 Critical conductance distribution of anisotropic system with spinorbit coupling ...................... 37

Figure 4.1 Critical conductance distribution of isotropic systems . . . . . 43

Figure 4.2 Distribution of lowest channel contributions . . . . . . . . . 46

Figure $4.3 \quad$ Fits to the critical distribution . . . . . . . . . . . . . 48

Figure $4.4 \quad$ Behavior at $g>1 \ldots \ldots \ldots \ldots$

Figure 5.1 Conductance fluctuations in 2D systems as a function of average conductance ...................... 56 
Figure 5.2 Conductance fluctuations in 2D systems as a function of disorder strength $\ldots \ldots \ldots \ldots \ldots \ldots \ldots \ldots$

Figure 5.3 Conductance fluctuations in $3 \mathrm{D}$ systems as a function of average

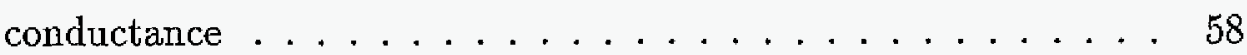

Figure 5.4 Conductance fluctuations in 3D systems as a function of disorder strength $\ldots \ldots \ldots \ldots \ldots \ldots \ldots \ldots$

Figure 5.5 Conductance fluctuations in systems with spin-orbit interactions 60

Figure A.1 Energy level statistics at lower band edge . . . . . . . 66

Figure A.2 Energy level statistics below band center $\ldots \ldots \ldots \ldots 7$

Figure A.3 Energy level statistics at band center $\ldots \ldots \ldots \ldots 8$

Figure A.4 Energy level statistics above band center . . . . . . . . . . 69

Figure A.5 Energy level statistics at upper band edge . . . . . . . . . 69

Figure B.1 Critical wavefunction of an isotropic system . . . . . . . 75

Figure B.2 Localized wavefunction of an isotropic system . . . . . . 76

Figure B.3 Critical wavefunction of an anisotropic square system $\ldots \ldots 77$

Figure B.4 Critical wavefunction of an anisotropic rectangular system . . . 78 


\section{LIST OF TABLES}

Table 4.1 Averages and variances of the conductance . . . . . . . . 44

Table $4.2 \quad$ Averages and variances of Lyapunov exponents . . . . . . . . . 47

Table 5.1 Universal conductance fluctuation values $\ldots \ldots \ldots 5$ 
I would like to thank Professor Soukoulis for the opportunity to work with him and obtain my degree under his guidance. Many thanks also go to Dr. Markoš for stimulating discussions, to the whole group for creating a pleasant working environment, and to the many friends I made, particularly the zeitgeist community, and who made my stay in Ames, the state of Iowa, and the United States a very positive experience. 


\section{CHAPTER 1. GENERAL INTRODUCTION}

Metal-to-insulator transitions are generally linked to two phenomena: electron-electron correlations and disorder. Although real systems are usually responding to a mixture of both, they can be classified as undergoing a Mott-transition, if the former process dominates, or an Anderson-transition, if the latter dominates. High- $T_{c}$ superconductors, e.g., are a candidate for the first class. Materials in which disorder drives the metalto-insulator transition include doped semiconductors and amorphous materials. After briefly reviewing the previous research on transport in disordered materials and the disorder-induced metal-to-insulator transition, a summary of the model and the methods used in subsequent chapters is given. This general introduction closes with an outline of the dissertation.

\subsection{Overview of transport in disordered media}

The field of transport in disordered systems in its present form owes much to a single paper, written by Anderson in 1958,[1] titled Absence of diffusion in certain random lattices. In this paper, Anderson discusses transport of (quasi-)particles by transitions between quantum-mechanical eigenstates of the system without thermal activation and the conditions under which such transport fails to extend beyond a finite subregion of the system. The particle is then said to be localized and the system is insulating, as there can be no transport of particles from one end of the sample to the other. If the system parameters can be controlled such that the conditions for localization are met for 
some range in parameter space, but not outside that range, the system can undergo a quantum phase transition from the localized, insulating phase to a conducting one, where eigenstates of particles extend throughout the system (even in the thermodynamical limit). Under the right conditions this can be achieved, e.g., by changing the Fermi energy of electrons in a crystal with point defects, or by changing the frequency of classical waves travelling through almost periodic structures, e.g., photonic band gap materials.[2] Typically, electron states are localized in the tails of the disorder-broadened bands, whereas the center of the bands is made up of extended states. The two regions are separated by a mobility edge, where the critical states of the metal-to-insulator transition are found.

Basically, there are two approaches for characterizing this transition: one that focuses on the wave function, working with the concepts of localized and extended states, and one that focuses on properties of the system, like conductance or resistance, working with the concepts of insulators and conductors. The former defines a length that is associated with the volume occupied by the wave function in a localized state and defines the transition to be at the point where this localization length diverges. The problem now lies in identifying the appropriate definition for this localization length. The second approach defines the critical state as the point where the conductance becomes independent of the system size, whereas it increases with system size for metallic systems and decreases with system size for insulating ones. As we are dealing with an ensemble of disordered systems and, therefore, a distribution of conductances, it becomes necessary to identify the single parameter, if it exists, that characterizes the full distribution of the conductance and controls the metal-to-insulator transition. Of course, both approaches must agree in their results when applied to a particular system.

During the 70 s and 80 s experiments were carried out, dealing with phenomena that show the existence of or can be explained with localized eigenstates of the systems involved and the corresponding quantum phase transition. Phonon-assisted hopping, 
e.g., can be used to explain[3] the low-temperature behavior of the conductance in amorphous semiconductors.[4] Bergmann[5] reports on weak localization in thin films resulting from quantum interferences. The Anderson transition was studied[6] on uncompensated doped semiconductors, e.g. Si:P, $[7]$ as well as compensated doped semiconductors and amporphous metals and semiconductors, e.g. $\mathrm{Al}_{\mathbf{x}} \mathrm{Ga}_{1-\mathbf{x}}$ As.[8] The former exhibited a critical exponent for the doping concentration of $1 / 2$, whereas the latter have a critical exponent for the charge carrier concentration of 1 , with some exceptions in both camps.[9] Theory expects a value of 1 in all those cases[10, 11] (considering 3D systems with orthogonal symmetry), whereas numerical calculations find values around 1.5.[12] Localized states are also a useful basis for understanding the integer Quantum Hall effect.[13] Another disorder-induced phenomenon, the socalled universal conductance fluctuations, was also discovered during that period.[14] Here, the variance of the conductance in disordered mesoscopic systems is constant over a finite range of average conductance and independent of the system size (as long as phase coherence is maintained throughout the sample). Localization of light and water waves follows the same general pattern.[15] In the 90s, efforts were made to understand the interplay between particle-particle interactions and disorder in driving the delocalization[16] after the observation of anomalously large persistent currents in mesoscopic metallic rings.[17] Shepelyansky[18] tackled the problem of two interacting particles in a strictly one-dimensional system and found that correlated pairs can have much larger localization lengths than non-interacting particles. Shortly thereafter, experiments indicated a metal-insulator transition in two-dimensional systerns at $T=0$ and $B=0,[19,20,21]$ which cannot be explained within the established framework of non-interacting particles. A wide variety of experimental and theoretical results on the interplay of disorder and interactions were presented at two conferences in 1998[22] and 1999.[23]

The theoretical efforts dealing with the metal-to-insulator transition center around the renormalization group theory and the one-parameter scaling approach.[24] In gen- 
eral, when describing a system within the renormalization group approach, one considers a set of parameters, depending on system size, the values of which for a size $b L$ are a function of only the parameters at size $L$ and the scale factor $b$. Of special interest are then the sets of fixed points, where all the parameters are independent of $b$ (and thus independent of $L$ as well), as phase transitions will occur at such points. In a region close to one fixed point, the renormalization function may be linearized. Restricting the investigation to these critical regions is the idea behind the scaling approach. Parameters are classified as relevant or irrelevant, depending on whether they are responsible for repulsion from or attraction to the critical point under consideration. That is, if all other parameters are set to their fixed point values, and repeated application of the renormalization function leads the system further away from the fixed point, the parameter is relevant. One-parameter scaling theories assume that there is only one relevant parameter. The assumption has been validated mostly through numerical applications.

The scaling approach predicted that metallic states, and thus a true metal-to-instlator transition, cannot be present in systems of non-interacting particles in one or two dimensions. However, as evidenced by the Quantum Hall effect, the application of a magnetic field perpendicular to the plane of a two-dimensional system may introduce extended states that contribute to the conductance of a sample. Also, the introduction of spin-orbit scattering into the model may result in a true metal-to-insulator transition in two dimensions. This difference in behavior can be understood from the random matrix theory, where systems are divided into three universality classes depending on whether time reversal or rotational symmetries are broken or not, and it allows the use of numerical methods, which are much more powerful in dealing with systems of lower dimensions due to the rapid increase in computational effort with system size in three-dimensional systems. Two-dimensional systems are used to calculate the conductance, the spectrum of eigenenergies, and wave functions, whereas the calculation of localization lengths requires quasi-one-dimensional systems which have a cross section 
much smaller than their length in the direction of transport. However, the inclusion of Coulomb-repulsion into the model (to study the competition with disorder in causing the transition) still limits numerical calculations mostly to either one-dimensional systems or rather small higher-dimensional ones.

Models employed in numerical studies include tight-binding models, including the Hubbard model which includes an on-site interaction between particles, real space models, the Chalker-Coddington network model[25] and derivatives, and, where magnetic fields are present, Landau-space models. Besides calculating conductances or localization lengths, some effort has been put into the examination of energy level spacing distributions, which are an indicator for the metal-to-insulator transition, and other properties of the energy eigenvalue spectrum, as well as the multifractal character of eigenfunctions at the critical point, which can be related to some scaling properties. Analytical methods include the application of a non-linear $\sigma$ type model and direct diagrammatical summation.

\subsection{Model and methods}

Throughout the work presented in this thesis the tight-binding model has been used. Its inherently discrete character makes it particularly easy to implement numerically. In this model, the Hamiltonian is expressed with respect to states that are essentially localized at one lattice site, usually atomic orbitals (or linear combinations) corresponding to the principal quantum number of the electron that is supposed to be disassociated from the atom and diffuse through the crystal. Assuming that overlap between orbitals centered at different lattice sites is so small that hopping is only allowed between nearest neighbor atoms, the energy eigenvalue bandwidth of such a Hamiltonian in square or cubic lattices is eight (2D) or twelve (3D) times the value of the overlap integral. Generally the model Hamiltonian is written 


$$
\mathcal{H}=\sum_{n}|n\rangle \varepsilon_{n}\left\langle n\left|+\sum_{m, n}^{\prime}\right| m\right\rangle V_{m, n}\langle n|
$$

where the ket $|n\rangle$ denotes a state centered at lattice site $\mathbf{R}_{n}, V_{m, n}$ the overlap integral and the primed sum runs only over lattice sites $m \neq n$. Without disorder, the site energies $\varepsilon_{n}$ are all equal and thus set to 0 . Disorder can be introduced by choosing these and/or the hopping integrals from a random distribution of values. This introduces allowable energy values slightly outside the band of the system without disorder and smoothes out a singularity of the density of states at the band center.

A magnetic field is introduced into the system by replacing the operator $\hbar \mathbf{k}$ with $\mathbf{p}-e \mathbf{A} / c$ (the socalled Peierls substitution), where $\mathbf{A}$ is the vector potential and $\nabla \times \mathbf{A}=$ B the magnetic induction. This leads to a rather complicated band structure[26] and phase factors in the hopping integrals. These phases contain the parameter $\alpha=e a^{2} B / h c$, with the lattice constant $a$. Thus, for $\alpha=1$, the rather large $a=0.5 \mathrm{~nm}$ translates into the extremely large $B=5 \mathrm{TT}=5 \cdot 10^{12} \mathrm{~T}(! !)$, but due to the number of bands being equal to the denominator in a fractional $\alpha$, too small values of $\alpha$ are numerically inadvisable. To include spin-orbit interactions, another quantum number, $\sigma$, is introduced, with the allowed values +1 and -1 . The hopping integrals turn into $2 \times 2$ matrices, while the site energies are usually kept independent of $\sigma$.

\subsubsection{Localization length}

To calculate the localization length, the transfer matrix method is employed. The system is cut into slices (in our case of thickness $a$ ) and the values of the wave function at the lattice sites in the first two slices are generated randomly. The transfer matrix is then applied iteratively to generate from the values in slices $n-1, n$ the new values in slices $n, n+1$. According to Oseledec's Theorem[27] the product of a large enough number of these transfer matrices converges to a matrix $T$, and eigenvalues of $\left(T^{\mathrm{t}} T\right)^{1 / 2}$ exist for 
almost all disorder configurations. The transfer matrices in our problem are symplectic, which means eigenvalues come in pairs $\nu_{i}, 1 / \nu_{i}$, or, equivalently, $\exp \left(N \gamma_{i}\right), \exp \left(-N \gamma_{i}\right)$. The index $i$ runs from 1 to the number $M$ of lattice sites in one slice, while $N \gg M$ denotes the number of slices in the system. To find more than just the one eigenvalue with the largest magnitude, one has to apply the procedure to several random starting vectors and keep them orthonormalized. The exponents $\gamma_{i}$ in the eigenvalues with magnitude less than 1 are a measure for how quickly a state deteriorates when "transferred" through the disordered system. The inverse of the smallest $\gamma_{i}$ is then defined to be the finite size localization length for this system

$$
\lambda_{M}=1 / \min _{i}\left\{\gamma_{i}\right\}
$$

These are calculated for different disorder configurations to get an ensemble average and then finite size scaling is applied to the ensemble averaged $\lambda_{M}$ for cross sections of different size $M$. The scaling function is $\lambda_{M} / M$ as a funtion of $\xi / M$, where the localization length $\xi$ is obtained from the large $M$ limit of $\lambda_{M}$. As both $\lambda_{M}$ and $\xi$ depend parametrically on disorder strength and fermi energy of the electron, the scaling function-and as a consequence $\xi$-can also be obtained by fitting a set of data for $\lambda_{M}(W, E)$ with varying values of $W$ and $E$ to a single curve. The form of the scaling function must be independent of these parameters if the one-parameter scaling theory works. In numerical calculations of the localization length this theory has generally been supported well by the data obtained.

\subsubsection{Spectra and eigenstates}

To calculate parts of or the whole spectrum of eigenvalues of a given Hamiltonian, it is advisable to employ methods that make use of the sparseness of the Hamilton matrix. As we are dealing with a Hermitian matrix, we can make a socalled Lanczos transformation to a real symmetric tridiagonal matrix of an order independent of the original matrix 
order. Increasing the size of the Lanczos matrix allows for an easy and numerically viable way to make up for numerical errors in the calculation of eigenvalues. In exact arithmetic, the Lanczos matrix of the same order as the Hamilton matrix will have exactly the same eigenvalues, i.e., in this case the Lanczos transformation is a unitary transformation of the Hamilton matrix. In numerical implementations, however, the method can not accurately predict the multiplicity of a given eigenvalue. Fortunately, in a disordered system, we can expect the probability for an eigenvalue to be degenerate to be so small as to be negligible, so that, as soon as a value appears to be numerically multiple, we can assume that it has converged to an eigenvalue of the Hamiltonian to within numerical precision.

The spectrum is then immediately available for analysis by established methods of random matrix theory[28] (for application to the case studied in Chapter 2, see Appendix A). Once the critical energy $E_{c}$ of the metal-to-insulator transition is known, the eigenvector of the Lanczos matrix to the eigenvalue closest to $E_{c}$ can be calculated easily and then backtransformed to find the critical eigenstate of the Hamiltonian, which in turn can be subjected to multifractal analysis[29] (see Appendix B).

\subsubsection{Conductance}

The Landauer formula[30] for a system with ideal leads is used as a basis for the conductance calculations:

$$
g=\operatorname{Tr}\left(t^{\dagger} t\right)
$$

with the transmission matrix $t$ determining the transmission of an electron through the sample. For numerical application the matrix $t^{\dagger} t$ has to be related to the transfer matrix $T$ via its transformation to "flux representation" $Q$ by (for details see Appendix C):

$$
4\left(Q Q^{\dagger}+\left(Q Q^{\dagger}\right)^{-1}+2 I\right)^{-1}=\left(\begin{array}{cc}
t^{\dagger} t & 0 \\
0 & t^{\prime} t^{\dagger \dagger}
\end{array}\right)
$$


The numerical implementation calculates therefore the eigenvalues $z_{j}$ of $Q Q^{\dagger}$ for a large ensemble of systems with different disorder configurations, then calculates the conductance $g$ as

$$
g=\sum_{j} g_{j}=\sum_{j} \frac{4}{z_{j}+z_{j}^{-1}+2}
$$

More efficient algorithms can be employed if only the trace $g$ is required instead of all the individual $g_{j}$.

\subsection{Dissertation Organization}

In Chapter 2, the critical behavior of two-dimensional anisotropic systems in the presence of a magnetic field is investigated, using the localization length and properties of the eigenvalue spectrum. The relationship of the scaling functions to that of isotropic systems is given. Having found the critical energy, we proceed in Chapter 3 to discuss the critical conductance distribution of such systems, again in reference to that of isotropic systems. The method is also applied to systems with spin-orbit interactions. Seeing a satisfying relationship between anisotropic systems and their isotropic counterparts, we focus in the remaining chapters on isotropic systems. As there is so far no complete description of the analytic form of the critical distribution of the conductance, in Chapter 4, a more extensive characterization of that distribution is carried out in isotropic systems of different universality classes. The numerical data is compared to some analytical results. Away from the critical point, on the other hand, a more interesting quantitiy to study is the variance of the conductance, especially in a range of metallic behavior where the magnitude of the variance is independent of the avarage of the conductance, i.e., in the range of universal conductance fluctuations. Chapter 5 contains a discussion of how the ensemble fluctuations of the conductance change as a function of disorder strength, including the ballistic, diffusive and critical/localized regimes. Particular emphasis is given to the influence of boundary conditions. In Chapter 6, some general 
conclusions are presented. In Appendix A, additional data about the spectral statistics of anisotropic unitary systems, used but not presented in the discussion in Chapter 2, is given. Appendix B has some results from multifractal analysis of the critical eigenstates of such systems. In Appendix C, the relation between the conductance $g$ and the transfer matrix $T$, as discussed above is provided in detail.

\section{Bibliography}

[1] P. W. Anderson, Phys. Rev. 109, 1492 (1958).

[2] For an overview see Photonic Band Gap Meterials, edited by C. M. Soukoulis, (Kluwer Academic Publishers, Dordrecht/Boston/London, 1995).

[3] H. Böttger and V. V. Bryksin, Hopping Conduction in Solids, (VCH Publishers, Deerfield Beach, FL, 1985).

[4] N. F. Mott and E. A. Davis, Electronic processes in non-crystalline materials, 2nd ed. (Clarendon Press, Oxford, 1979).

[5] G. Bergmann, Phys. Rep. 107, 1 (1984).

[6] for a detailed discussion see, e.g., G. A. Thomas, in Localisation and interaction in disordered metals and doped semiconductors, edited by D. M. Finlayson (SSUSP, Edinburgh, 1986), p. 172.

[7] M. A. Paalanen and G. A. Thomas, Helv. Phys. Acta 56, 27 (1983).

[8] S. Katsumoto, F. Komori, N. Sano, and S. Kobayashi, J. Phys. Soc. Japan 56, 2259 (1987).

[9] see, e.g., M. P. Sarachik, in Metal-insulator transitions revisited, edited by P. P. Edwards and C. N. R. Rao (Taylor \& Francis, Bristol, PA, 1995), p. 79. 
[10] D. Vollhardt and P. Wölfle, Phys. Rev. Lett. 48, 699 (1982).

[11] F. J. Wegner, in Localization, Interaction, and Transport Phenomena, Springer Series in Solid State Sciences, Vol. 61, edited by B. Kramer, G. Bergmann, and Y. Bruynseraede (Springer, Berlin, 1985), p. 99.

[12] A. MacKinnon, J. Phys.: Condens. Matter 6, 2511 (1994).

[13] K. v. Klitzing, in Nobel Lectures in Physics v. 6, edited by S. Lundqvist (World Scientific, Singapore, 1992).

[14] C. P. Umbach, S. Washburn, R. B. Laibowitz, and R. A. Webb, Phys. Rev. B 30, 4048 (1984); S. Washburn and R. A. Webb, Adv. Phys. 35, 375 (1986).

[15] Scattering and localization of classical waves in random media, edited by $\mathrm{P}$. Sheng (World Scientific, Singapore, 1990).

[16] see, e.g., A. Muller-Groeling, H. A. Weidenmuller, and C. H. Lewenkopf, Europhys. Lett. 22, 193 (1993); M. Abraham and R. Berkovits, Phys. Rev. Lett. 70, 1509 (1993); G. Bouzerar, D. Poilblanc, and G. Montambaux, Phys. Rev. B 49, 8258 (1994).

[17] L. P. Lévy, G. Dolan, J. Dunsmuir, and H Bouchiat, Phys. Rev. Lett. bf 64, 2074 (1990)

[18] D. L. Shepelyansky, Phys. Rev. Lett. 73, 2607 (1994).

[19] S. V. Kravchenko, W. E. Mason, G. E. Bowker, J. E. Furneaux, V. M. Pudalov, and M. D'Iorio, Phys. Rev. B 51, 7038 (1995); S. V. Kravchenko, D. Simonian, M. P. Sarachik, W. Mason, and J. E. Furneaux, Phys. Rev. Lett. 77, 4938 (1996).

[20] D. Popović, A. B. Fowler, and S. Washburn, Phys. Rev. Lett. 79, 1543 (1997). 
[21] M. Y. Simmons, A. R. Hamilton, M. Pepper, E. H. Linfield, P. D. Rose, D. A. Ritchie, A. K. Savchenko, and T. G. Griffiths, Phys. Rev. Lett. 80, 1292 (1998).

[22] Proc. 210. WE-Heraeus Seminar on Percolation, Interaction, Localization: Simulations of Transport in Disordered Systems, Berlin, edited by M. Schreiber, Ann. Phys. (Leipzig) 5-6 (1998).

[23] Proc. Int. Conf. LOCALIZATION 1999: Disorder and Interaction in Transport Phenomena, Hamburg, edited by M. Schreiber, Ann. Phys. (Leipzig) 7-9 (1999).

[24] R. J. Creswick, Ha. A. Farach, and C. P. Poole, Jr., Introduction to renormalization group methods in physics (Wiley-Interscience, New York, NY, 1992).

[25] J. T. Chalker and P.D. Coddington, J. Phys. C 21, 2665 (1988).

[26] D. R. Hofstadter, Phys. Rev. B 14, 2239 (1976).

[27] V. I. Oseledec, Trans. Moscow Math. Soc. 19, 197 (1968).

[28] M. L. Mehta, Random Matrices, Second edition, (Academic Press, San Diego, 1991); O. Bohigas and M.-J. Giannoni, in Mathematical and Computational Methods in Nuclear Physics, Lecture Notes in Physics, Vol. 209 (Springer, New York, 1984), pp. 1-99.

[29] For an overview see M. Janssen, Int. J. Mod. Phys. B 8, 943 (1994); M. Janssen, Phys. Rep. 295, 1 (1998).

[30] R. Landauer, IBM J. Res. Dev. 1, 223 (1957); E. N. Economou and C. M. Soukoulis, Phys. Rev. Lett. 46, 618 (1981); D. S. Fischer and P. A. Lee, Phys. Rev. B 23, $6851(1981)$. 


\title{
CHAPTER 2. METAL-INSULATOR TRANSITIONS IN ANISOTROPIC TWO-DIMENSIONAL SYSTEMS
}

\author{
A paper published in Physical Review B 63, $085103(2001)^{1}$ \\ and online at cond-mat/0010430
}

Marc Rühländer and C. M. Soukoulis

\section{Abstract}

Several phenomena related to the critical behavior of noninteracting electrons in a disordered two-dimensional tight-binding system with a magnetic field are studied. Localization lengths, critical exponents and density of states are computed using transfermatrix techniques. Scaling functions of isotropic systems are recovered once the dimension of the system in each direction is chosen proportional to the localization length. It is also found that the critical point is independent of the propagation direction, and that the critical exponents for the localization length for both propagating directions are equal to that of the isotropic system, $\nu \approx 7 / 3$. We also calculate the critical value $\Lambda_{c}$ of the scaling function for both the isotropic and the anisotropic system. It is found that $\Lambda_{c}^{\text {iso }}=\sqrt{\Lambda_{c}^{x} \cdot \Lambda_{c}^{y}}$. Detailed numerical studies of the density of states $n(E)$ for the isotropic system reveals that for an appreciable amount of disorder, the critical energy is off the band center.

\footnotetext{
${ }^{1}$ (C)2001 The American Physical Society
} 


\subsection{Introduction}

The problem of Anderson localization[1] in anisotropic sysytems has attracted considerable attention $[2,3,4,5]$ recently. It is generally accepted[2] that anisotropy does not change the universality class and that the isotropic results are recovered once a proper scaling of the anisotropic results is performed. If the dimension of the system size is chosen to be directly proportional to the localization length, the system should be effectively isotropic. The difficulty in implementing such a procedure lies in the fact that the localization lengths are usually not known a priori. It was found through detailed numerical calculations[2] that this scaling indeed works. It was also shown[6] that the probability distributions of the conductance in the two directions are exactly equal to each other, provided that the ratio of the sides of the rectangle is proportional to the ratio of the localization lengths in the two directions. These scaling results were obtained for an anisotropic system where all the states were localized.

It is well known[1] that noninteracting electrons are localized in two-dimensional (2D) disordered systems. There are, however, some exceptions to this rule. These include electrons having strong spin-orbit coupling, [7] integer quantum Hall systems, [8] and tight-binding models with random magnetic fields.[9] The best known example is the integer quantum Hall plateau transition occurring in a $2 \mathrm{D}$ noninteracting system in a strong magnetic field. Extended states do not exist as a result of Anderson localization except at a singular energy near the center of each of the Landau subbands. $[8,10]$ The localization length diverges at these critical energies $E_{c}$ as $\xi \propto\left|E-E_{c}\right|^{-\nu}$.

Another important point is the universality of the conductance at the critical point of the Anderson transition for the anisotropic system.[4] From the generalized scaling functions, it has been established that the geometric mean of the critical value $\Lambda_{c}$ of the scaling function $\Lambda \stackrel{\text { def }}{=}\left(\lambda_{M} / M\right)$ (as a function of $\left.\xi / M\right)$ is a constant independent of the strength of the anisotropy. (Here $\lambda_{M}$ denotes the finite-size localization length of a 
quasi-one-dimensional strip of finite width $M$.) Numerical calculations in both two-[2] and three-dimensional[4] disordered anisotropic systems support this claim. However, the same is not true for the conductance. Numerical calculations[4] in three-dimensional anisotropic systems do not support a universal value of the conductance for the geometric mean. This might be due to too small sizes used in the 3D system or to a lack of universality of the conductance at the critical point.

In this paper, we investigate the scaling properties of the finite-size localization length $\lambda_{M}$ and the critical value $\Lambda_{c}$ of the scaling function in a two-dimensional system described by a tight-binding model in the presence of a magnetic field. Both the isotropic case as well as the anisotropic case will be examined. This is perhaps the simplest system that exhibits the correct behavior of the metal-to-insulator transition. To our knowledge, no such calculations have been previously reported for the anisotropic tight-binding model with a constant magnetic field. Some of the questions we try to answer are as follows: How does the anisotropy affect the critical behavior, especially, will there be one or two critical exponents for the localization lengths? How do the anisotropic quantities relate to the corresponding isotropic ones, especially, can we expect the geometric mean of the two anisotropic values to equal the isotropic value? What are the values for the scaling functions at the critical point? In Sec. 2.2, we describe the model and the numerical methods we used. In Sec. 2.3, we present and discuss our numerical results. In Sec. 2.4, we summarize the conclusions of this work.

\subsection{Model and Methods}

In the tight-binding model, one has the Hamiltonian

$$
\mathcal{H}=\sum_{i}|i\rangle \varepsilon_{i}\left\langle i\left|+\sum_{i, j}^{\prime}\right| i\right\rangle V_{i j}\langle j|
$$

where the summations run over lattice sites $i$ and $j$. We consider only nearest-neighbor 
interaction in the hopping integrals $V_{i j}$. The effects of an external magnetic field, characterised by a vector potential $\mathbf{A}(\nabla \times \mathbf{A}=\mathbf{B})$, enter the model via phases of the hopping integrals with

$$
V_{i j}=t_{i j} e^{-2 \pi i(e / h) \int_{\mathbf{r}_{i}}^{\mathbf{r}_{j}} \mathbf{A}(\mathbf{r}) \mathrm{dr}}
$$

the integral connecting lattice sites $i$ and $j$ by a straight line. In two dimensions with a magnetic induction $\mathbf{B}$ perpendicular to the plane of the system, one can choose the gauge for the vector potential in such a manner that the phases vanish in one direction within the plane and are integer multiples of some number $2 \pi \alpha$ in the other direction, such that the value of $\alpha$ completely characterizes the influences of the magnetic field on the system. In particular, the denominator of a rational $\alpha$ equals the number of bands in the density of states of the system without disorder. Introducing anisotropy into the system by choosing different amplitudes $t_{i j}$ in the two directions within the plane will effect only the position of these bands, not their number. We bring disorder into the system by independently choosing all the site energies $\varepsilon_{i}$ from a rectangular distribution of width $W$ centered at 0 ; thus $W$ is a measure of disorder strength. Both $W$ and $E$ are measured in units of the largest hopping matrix element $t$, which is taken to be unity.

As our main method we use the transfer matrix method,[1] where a matrix $\mathrm{T}_{M}$ connects the amplitudes of a state at both ends of a quasi-one-dimensional strip of width $M$ and length $N \gg M$. Due to the anisotropy, we have to do this in the two spatial directions seperately. Therefore, we get two sets of parameters $\lambda_{M, x}$ and $\lambda_{M, y}$, which lead to two seperate localization lengths $\xi_{x}$ and $\xi_{y}$ in the $x$ and $y$ direction, respectively. Scaling of the data is used to improve on the values of $\xi_{x}$ and $\xi_{y}$, which are then analyzed to find the critical energies, where the localization lengths diverge as well as the critical exponents of these divergences.

We obtain the density of states by using a Lanczos procedure[11] to diagonalize 
the Hamiltonian on squares of (linear) size $L$. The energy-level separation distribution function $p(s)$ should deviate markedly from a Poisson distribution for the local density of states around the critical energy, approaching the Wigner distribution for the unitary ensemble. $[12,13]$ We also use the density of states to show that for sufficiently strong disorder the critical energy does not necessarily coincide with the band center.

\subsection{Results}

To obtain the critical energy $E_{c}$ for the anisotropic tight-binding model, first we calculate $\lambda_{M, x}$ and $\lambda_{M, y}$ for different strip widths $M$ and energies $E$ above and below the critical energy $E_{\mathrm{c}}$. As the exact position of $E_{c}$ varies with the disorder strength $W$, the hopping integral $t_{x}<1$ in the difficult hopping direction as well as the magnetic field parameter $\alpha$, we restrict our investigation to one set of these parameters $W=0.1$, $t_{x}=0.8$ and $\alpha=\frac{1}{8}$. The data for the more localised states show that $M / \lambda_{M}$ versus $M$ is a straight line. The inverse slope of each of these lines gives a first estimate for the localization lengths $\xi_{x}$ or $\xi_{y}$, respectively, thus the smaller the slope, the more extended are the corresponding eigenstates of the system. For energies closer to $E_{c}$ the lines would be essentially horizontal. In order to accurately obtain $E_{c}$, we have systematically calculated $\lambda_{M, x}$ and $\lambda_{M, y}$ for large $M$. The results are shown in Fig. 2.1, where we plot $\lambda_{M, x}$ and $\lambda_{M, y}$ for the anisotropic case for energies very close to $E_{c}$. From Fig. 2.1 we can confirm the existance of an extended state. Notice that $\lambda_{M} / M$ decreases as a function of $M$, which signifies localized states. For localized states, $\lambda_{M}$ eventually reaches its large$M$ limit, which is a constant, and therefore $\lambda_{M} / M$ decreases as $M$ increases. However, as can be seen from Fig. 2.1, at the critical energy $E_{c}, \lambda_{M} / M$ saturates to a constant due to the absence of length scales. For the case studied $\left(W=0.1, t_{y}=1.0, t_{x}=0.8\right.$, and $\alpha=\frac{1}{8}$ ), we find that the critical energy $E_{c}$ is between -2.966 and -2.965 , but closer to the second value. From Fig. 2.1, we can also obtain the critical values $\Lambda_{c}$ of $\lambda_{M} / M$ 

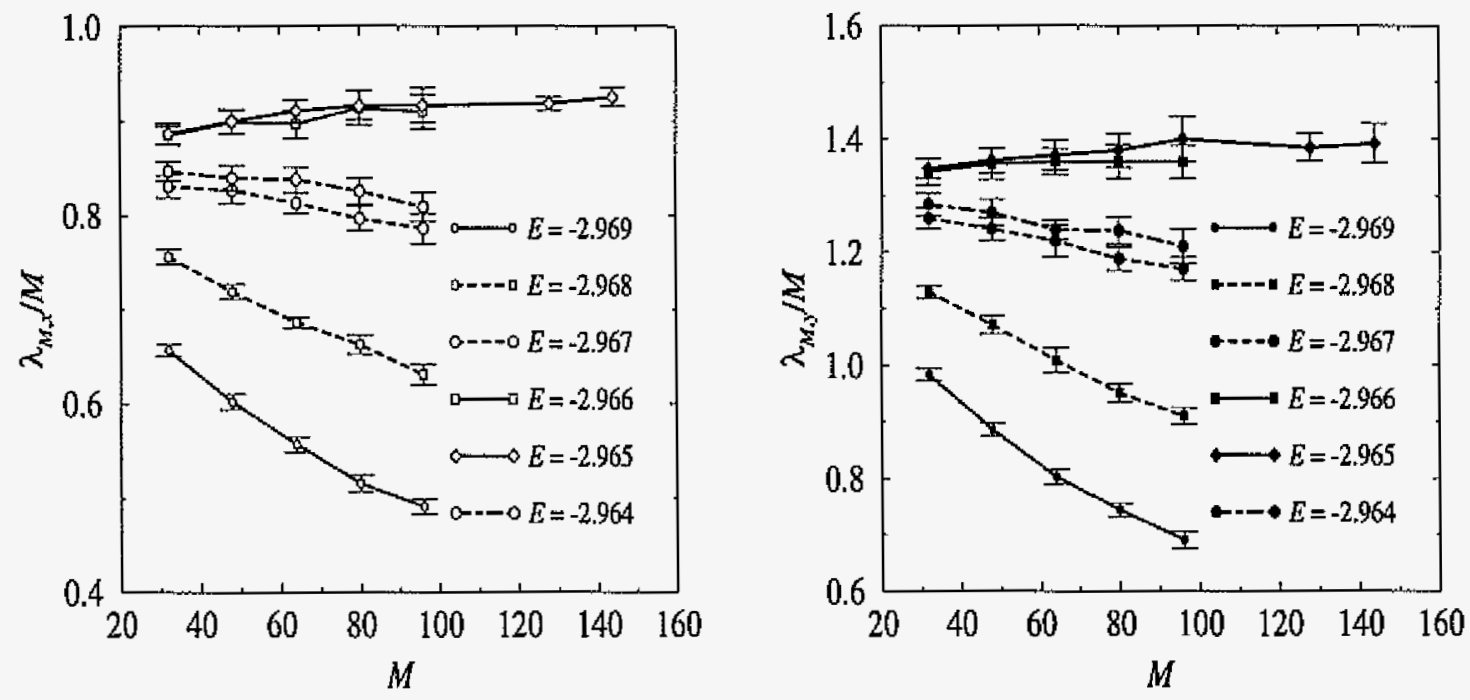

Figure 2.1 The critical values of the scaling functionns can be obtained from the large- $M$ limit of $\lambda_{M} / M$ at the critical energy. From the almost symmetrical behaviour of the values for the non-critical energies at either side of the critical one, we assume that the value for $E_{c}$ is between -2.966 and -2.965 , but closer to the second one. Left: difficult hopping direction; right: easy hopping direction.

for both directions of propagation. We find $\Lambda_{c}^{x}=0.92 \pm 0.01$ and $\Lambda_{c}^{y}=1.39 \pm 0.01$ with a geometric mean of $1.13 \pm 0.01$.

To confirm that the geometric mean $\Lambda_{c}$ of the two anisotropic values is related to the value for the isotropic case, we have calculated systematically $\lambda_{M} / M$ versus $M$ for the isotropic system $\left(W=4.0, t_{x}=t_{y}=1.0\right.$, and $\alpha=\frac{1}{8}$ ) for very large values of $M$. These results are shown in Fig. 2.2. From Fig. 2.2, we obtain that indeed $E_{c}=-3.40$ in this case, in agreement with previous results[10,14] that used different techniques to get $E_{c}$. In addition, Fig. 2.2 shows clearly that at the critical point of the isotropic system $\Lambda_{c}^{\text {iso }}=1.10 \pm 0.03$, which is approximately equal to the geometric mean of the two anisotropic values $\Lambda_{c}^{x}$ and $\Lambda_{c}^{y}$.

The critical value of $\lambda_{M} / M$ is related to the exponent $\alpha_{0}$ that can be obtained from 


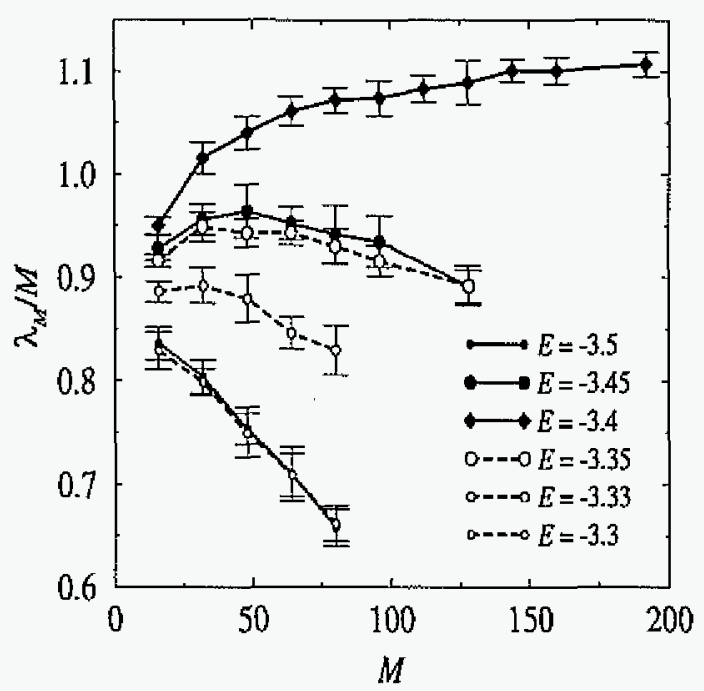

Figure 2.2 The critical value of the isotropic scaling function. From the large- $M$ data we estimate it to be $1.10 \pm 0.03$.

the multifractal analysis[8] of the eigenfunctions at the critical energy by $\Lambda_{c}^{-1}=\pi\left(\alpha_{0}-d\right)$ where $d$ is the Euclidian dimension of the system. Huckestein[26] calculated $\Lambda_{c}=1.14 \pm$ 0.02 for a real-space model, while Lee et al.[27] determine $\Lambda_{c}=\ln ^{-1}(1+\sqrt{2}) \approx 1.13$ for a network model, both of which are close to the value $\Lambda_{c}^{\text {is } o}=1.10 \pm 0.03$ obtained for the isotropic case of the 2D tight-binding model with a constant magnetic field.

The next step is to use the values for the localisation lengths obtained in this manner to plot $\lambda_{M, x} / M$ as a function of $\xi_{x} / M$ and $\lambda_{M, y} / M$ as a function of $\xi_{y} / M$. After combining the data for all energies into one graph, one usually has to adjust the values for the localization lengths slightly to make the data fall on a smooth curve. Figure 2.3 shows that these two functions are independent of the value of $E$, as expected for oneparameter scaling. However, the two scaling functions differ in their large- $\xi$ limit: the value is higher for the easy-hopping direction. To compensate for this anisotropy effect we use the following straightforward idea:[2] $\lambda_{M, x}\left(\lambda_{M, y}\right)$ is a length in the $x(y)$ direction 


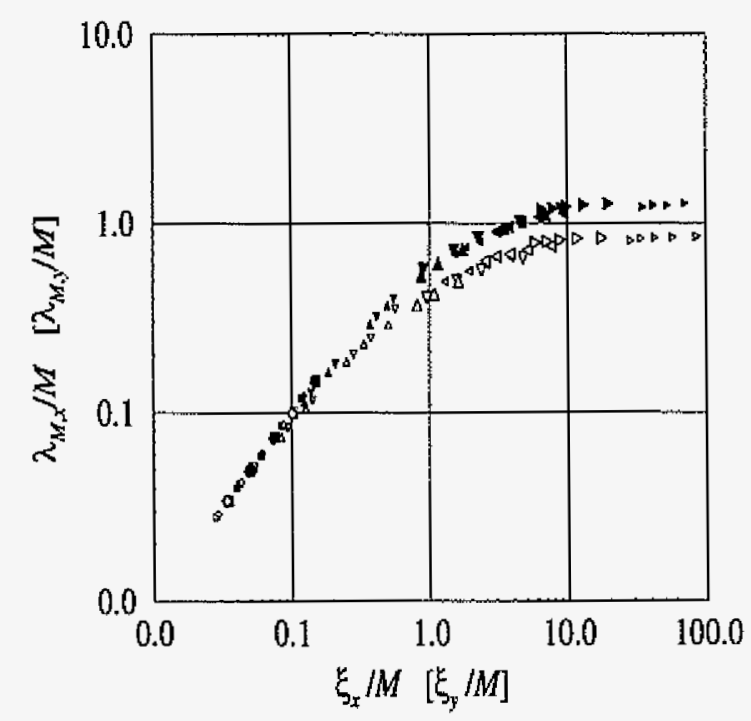

Figure 2.3 The scaling functions for the difficult- (open symbols) and easy(filled symbols) hopping directions $\lambda_{M} / M$ as a function of $\xi / M$. The localization lengths have been adjusted to better fit the data to a smooth curve. Energies are $-3.0,-2.99,-2.98$, $-2.97,-2.969,-2.968,-2.967,-2.964,-2.96,-2.95,-2.94$ and -2.93 .

along the length of the strip, so the appropriate scale should be $\xi_{x}\left(\xi_{y}\right)$. However, $M$ is a length measuring the width of the strip and therefore has to be scaled with the other localization length. Thus we plot $\left(\lambda_{M, x} / \xi_{x}\right)\left(\xi_{y} / M\right)$ vs $\left(\xi_{y} / M\right)$ and $\left(\lambda_{M, y} / \xi_{y}\right)\left(\xi_{x} / M\right)$ vs $\left(\xi_{x} / M\right)$ in Fig. 2.4. Not only do we obtain the same scaling function for both, but it is also the same as the isotropic one which we included for reference. The isotropic case was for $W=4$ and $\alpha=\frac{1}{8}$. Of course, under the assumption of one parameter scaling, the form of the (isotropic) scaling function should not depend on the values of $W$ and $\alpha$ directly (as long as neither vanishes completely) but only parametrically via the localization length $\xi(E, W, \alpha)$. Thus, the product of the two rescaled anisotropic functions equals the square of the isotropic scaling function. Immediately it is seen from this that the isotropic scaling function equals the geometric mean of the two anisotropic scaling 


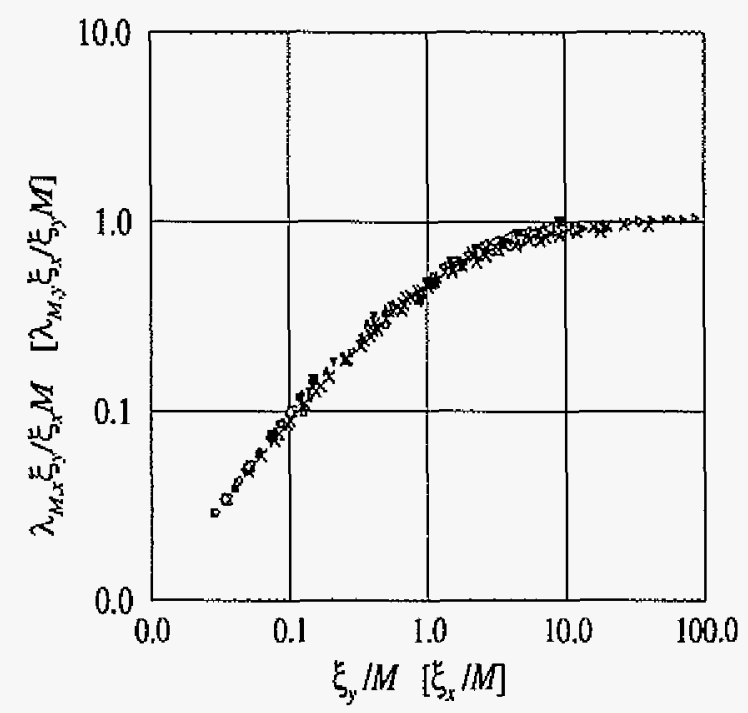

Figure 2.4 Plotting the rescaled scaling functions (cf. text) for the difficult(open symbols) and easy- (filled symbols) hopping directions together with the scaling function for an isotropic system (crosses).

functions,

$$
\left(\frac{\lambda_{M}}{M}\right)_{\text {iso }}=\sqrt{\frac{\lambda_{M, x}}{M} \cdot \frac{\lambda_{M, y}}{M}}
$$

as the rescaling factors $\xi_{x}$ and $\xi_{y}$ cancel each other. As we have shown before in Fig. 2.1 and Fig. 2.2, indeed Eq. (2.3) is obeyed.

The procedure of fitting the data to a smooth scaling function provides us with more accurate estimates of the localization lengths, which we can now use to determine the critical behaviour of $\xi$. In Fig. 2.5, we plot the localization lengths as a function of energy. One can clearly see that the states are less localized in the easy hopping direction, as was to be expected. Figure 2.5 also allows us to estimate $E_{c}$, the energy where the localization length diverges. We expect this critical energy to be independent of the strip orientation, as a higher-dimensional system would undergo a phase transition at this point, and our data give a strong indication that $E_{c}$ is indeed the same for both 


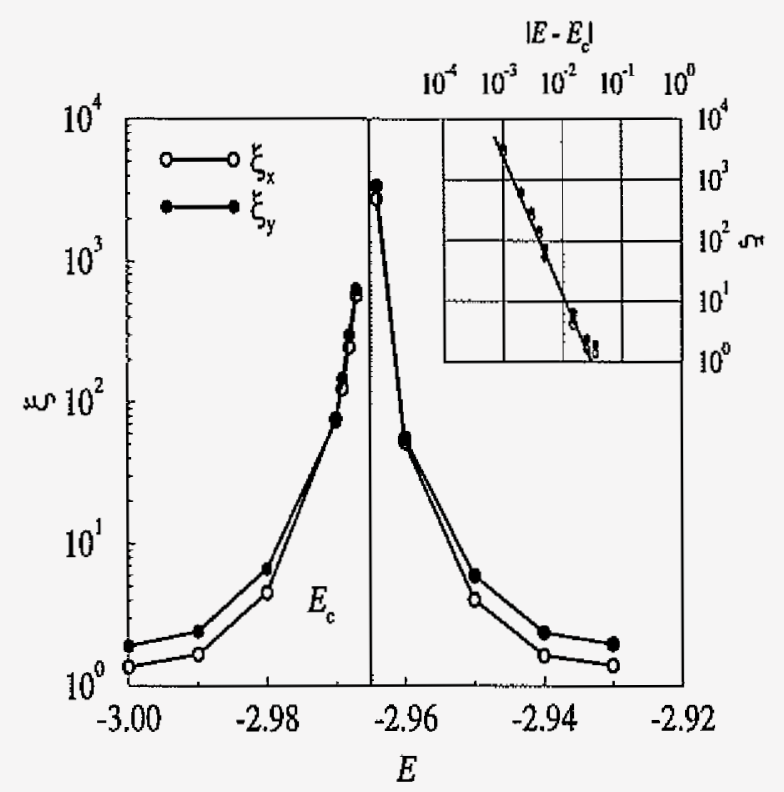

Figure 2.5 The divergence of the localization lengths at the critical energy; open symbols, difficult-hopping direction; filled symbols, easy-hopping direction. The values are taken after the adjustments made to obtain Fig. 2.4. Inset: To extract the critical exponent of the localization lengths we plot $\xi$ vs $\left|E-E_{c}\right|$ in a $\log -\log$ plot. Both exponents are found to be $2.3 \pm 0.1$, roughly equal to the theoretical value for the isotropic system.

directions. We estimate $E_{c} \approx-2.965 \pm 0.001$. This is consistent with the results obtained in Fig. 2.1.

The divergence of the localization length near the critical energy is expected to follow a power law

$$
\xi(E)=\xi_{0}\left|E-E_{c}\right|^{-\nu}
$$

with some critical exponent $\nu$. To test this hypothesis, we plot the logarithm of $\xi$ vs the logarithm of $\left|E-E_{c}\right|$. The result is shown in the inset of Fig. 2.5. That our data follows a straight line rather reasonably reconfirms our estimate for $E_{c}$, as the plot obviously is quite sensitive to the choice for that value. Furthermore, both sets of data can be 
fitted by the same straight line, giving the same critical exponent $\nu \approx 2.3 \pm 0.1$. Once again, this is the same as the isotropic value and very close to the theoretically predicted value[8] of $7 / 3$ for the isotropic system.

The distribution of energy-level separations in a given energy interval depends on the typical extension of the eigenstates of the system with eigenvalues in that energy region. Spatial overlap of eigenfunctions close in energy helps to delocalize the particle. In a finite system, more of the strongly localized eigenfunctions can be accomodated without significant overlap. The more extended the eigenfunctions become, the more difficult it becomes to fit several into the finite space, and they must be seperated in energy. This leads to the phenomenon of level repulsion, known from chaos theory. The corresponding distribution of level separations $s_{i}=E_{i}-E_{i-1}$ goes to zero for small s. In contrast, the distribution for a range of localized eigenstates has a maximum at vanishing level separation. More specifically, random matrix theory predicts[13] a Poisson distribution for the localized case and a Wigner distribution for the extended case. We have calculated the distribution of energy-level separations $p(s)$ for the anisotropic system studied in Fig. 2.1. We find that for an energy range close to $E_{c}=-2.965, p(s)$ is Wigner-like, whereas for the other energy ranges it is Poisson-like.

Level statistics for the isotropic system have been extensively studied by Potempa et al. $[14,15]$ and Batsch et al., $[16,17]$ proving the validity of the approach in distinguishing localized from extended states. In addition, the level number variance $\Sigma^{2}(\langle N\rangle)=\chi\langle N\rangle$ has been numerically obtained for the isotropic system,[18] using the Chalker-Coddington network model,[19] and compared with analytical theories,[20] which give for the spectral compressibilty $\chi=(d-D(2)) / 2 d$, where $D(2)$ is the multifractal exponent of the wavefunction at the critical point.[21] Klesse and Metzler obtain $\chi=0.124 \pm 0.006$.[18] Numerically obtained values for $D(2)$ include $1.43 \pm 0.03$ for a continuum model,[22] 1.56 for a network model,[23] and $1.62 \pm 0.02$ and 1.71 for a tight-binding model.[24, 25] Due to the limited size of our systems we were not able 

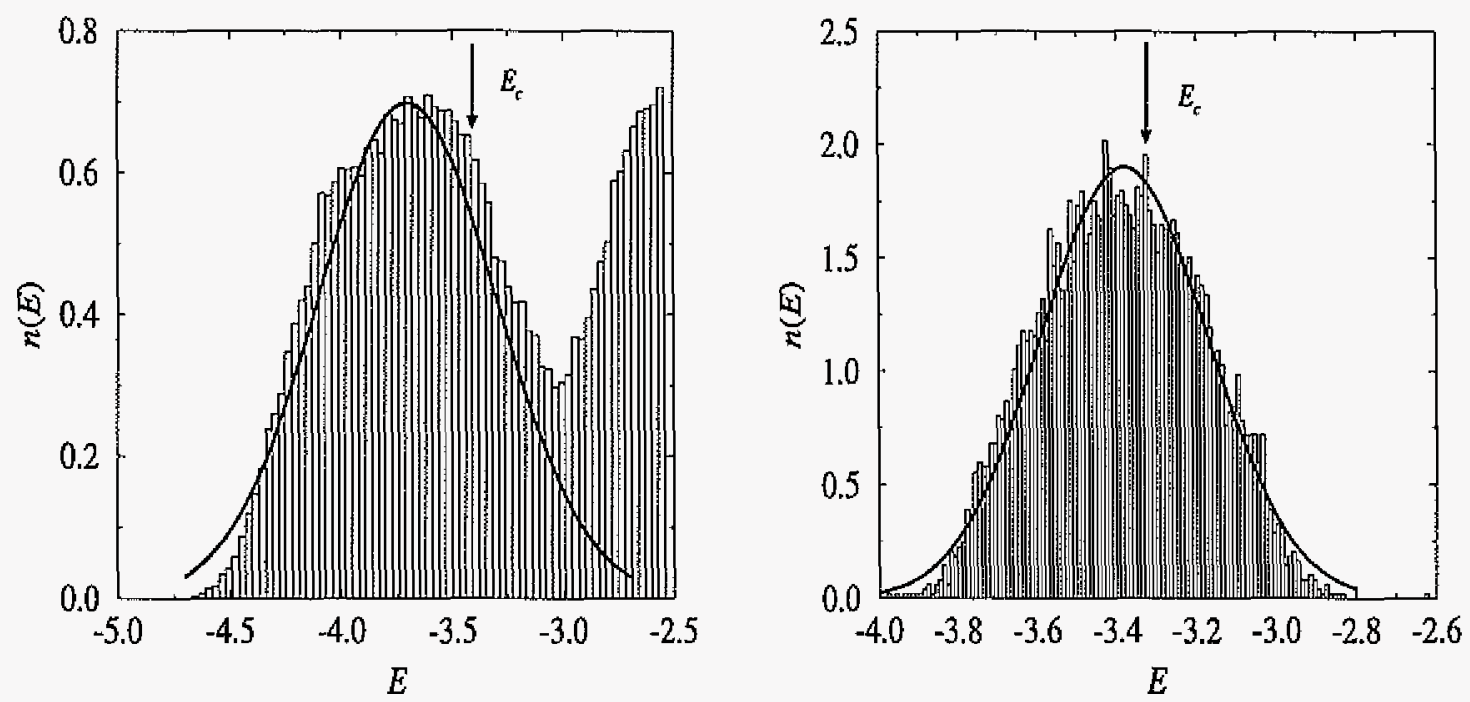

Figure 2.6 The density of states for the lowest subband for a disorder strength of $W=4.0$ (left) and $W=2.0$ (right), indicating that the critical energy is off the band center. A fit to a Gaussian distribution suggests that the band center in the $W=2.0$ case is at $E_{0} \approx-3.38$, whereas the critical energy is $E_{c} \approx-3.32$.

to produce results for $\chi$ for our anisotropic model. The number of energy eigenvalues sufficiently close to the critical point is not large enough to give good statistics for the number variance $\Sigma^{2}(\langle N\rangle)$. This point has to be adressed in the future.

Finally, Fig. 2.6 shows the positions of $E_{c}$ for isotropic systems at $W=2$ and $W=4$ to be different from the band center. Although a Gaussian is not the correct form for the density of states it is usually a reasonable fit. For the stronger disorder, $W=4$, the best approximation is achieved with a Gaussian centered at $E=-3.7$ with a standard deviation of $\sigma=0.4$. Figure 2.2 strongly indicates $E_{c}=-3.4$ (arrow in left panel of Fig. 2.6). Similarly, for the lesser disorder, $W=2$, a Gaussian centered at $E=-3.38$ with a standard deviation of $\sigma=0.21$. A plot similar to that for the more strongly disordered case shows that $E_{c}=-3.32$. However, for $W \leq 1$ the critical energy $E_{c}$ lies at the center of the Landau band. 


\subsection{Conclusions}

In summary, we have performed detailed numerical study of the scaling properties of highly anisotropic systems in 2D, with a metal-to-insulator transition. Scaling functions of the isotropic systems are recovered once the dimension of the anisotropic system is chosen to be proportional to the localization length. It is also found that the critical point is independent of the propagation direction and that the critical exponents for the localization length in both propagating directions are equal to that of the isotropic system. The critical value $\Lambda_{c}$ of the scaling function for both the isotropic and the anisotropic cases has been calculated. It is obtained that $\Lambda_{c}^{\text {iso }}=\sqrt{\Lambda_{c}^{x} \Lambda_{c}^{y}}=1.10 \pm 0.03$. Finally, density of states calculations revealed that the critical energy lies away from the center of the Landau band.

\section{Acknowledgements}

Ames Lraboratory is operated for the U.S. Department of Energy by Iowa State University under Contract No. W-7405-Eng-82. This work was supported by the Director for Energy Research, Office of Basic Sciences.

\section{Bibliography}

[1] For a recent review, see B. Kramer, and A. McKinnon, Rep. Prog. Phys. 56, 1469 (1993).

[2] Qiming Li, S. Katsoprinakis, E. N. Economou, and C. M. Soukoulis, Phys. Rev. B 56, R4297 (1997), and references therein.

[3] F. Milde, R. A. Römer, and M. Schreiber, Phys. Rev. B 55, 9463 (1997). 
[4] I. Zambetaki, Q. Li, E. N Economou, and C. M. Soukoulis, Phys. Rev. Lett. 76, 3614 (1996); I. Zambetaki, Q. Li, E. N. Economou, and C. M. Soukoulis, Phys. Rev. B 56, $12221(1997)$.

[5] N. Dupuis, Phys. Rev. B 56, 9377 (1997); C. Mauz, A. Rosch, and P. Wölfle, ibid. 56, 10953 (1997).

[6] X. Wang, Ph. D. thesis, lowa State University (1998); X. Wang, Q. Li, and C. M. Soukoulis (unpublished).

[7] S. Hikami, Prog. Theor. Phys. 63, 707 (1980).

[8] For a review, see B. Huckestein, Rev. Mod. Phys. 67, 357 (1995).

[9] A. Furusaki, Phys. Rev. Lett. 82, 604 (1999).

[10] X. Wang, Q. Li, and C. M. Soukoulis, Phys. Rev. B 58, 3576 (1998).

[11] J. K. Cullum, and R. A. Willoughby, Lanczos Algorithms for Large Symmetric Eigenvalue Computations (Birkhäuser, Boston, 1985), Vol. 3.

[12] O. Bohigas and M.-J. Giannoni, Mathematical and Computational Methods in Nuclear Physics, Lecture Notes in Physics Vol. 209 (Springer, New York, 1984), pp. $1-99$.

[13] M. L. Mehta, Random Matrices, Second edition, (Academic Press, San Diego, 1991).

[14] H. Potempa, A. Bäker, and L. Schweitzer, Physica A 256-258, 591 (1998).

[15] H. Potempa and L. Schweitzer, Ann. Phys. (Leipzig) 7, 457 (1998).

[16] M. Batsch and L. Schweitzer, Proceedings of the 12th Conference on High Magnetic Fields in the Physics of Semiconductors II, Würzburg 1996, (World Scientific, Singapore, River Edge, NJ, 1997), pp. 47-50. 
[17] M. Batsch, L. Schweitzer, and B. Kramer, Physica B 249-251, 792 (1998).

[18] R. Klesse and M. Metzler, Phys. Rev. Lett. 79, 721 (1997).

[19] J. T. Chalker and P.D. coddington, J. Phys. C 21, 2665 (1988).

[20] J. T. Chalker, V. E. Kravtsov, and I. V. Lerner, Pis'ma Zh. Éksp. Teor. Fiz. 64, 355 (1996) [JETP Lett. 64, 386 (1996)].

[21] For an overview see M. Janssen, Int. J. Mod. Phys. B 8, 943 (1994).

[22] W. Pook and M. Janssen, Z. Phys. B: Condens. Matter 82, 295 (1991).

[23] B. Huckestein and R. Klesse, Philos. Mag. B 771181 (1998).

[24] B. Huckestein and L. Schweitzer, High Magnetic Fields in Semiconductor Physics $I I I$, Springer Series in Solid-State Sciences Vol. 101, (Springer, Berlin, New York, 1992), pp. 84-88.

[25] B. Huckestein and L. Schweitzer, Phys. Rev. Lett. 72, 713 (1994).

[26] B. Huckestein, Phys. Rev. Lett. 72, 1080 (1994).

[27] D. H. Lee, Z. Wang, and S. Kivelson, Phys. Rev. Lett 70, 4130 (1993). 


\title{
CHAPTER 3. PROBABILITY DISTRIBUTION OF THE CONDUCTANCE IN ANISOTROPIC SYSTEMS
}

\author{
A paper published in Physical Review B 64, $193103(2001)^{1}$ \\ and online at cond-mat/0103594
}

Marc Rühländer, Peter Markoš, and C. M. Soukoulis

\begin{abstract}
We investigate the probability distribution $p(g)$ of the conductance $g$ in anisotropic two-dimensional systems. The scaling procedure applicable to mapping the conductance distributions of localized anisotropic systems to the corresponding isotropic one can be extended to systems at the critical point of the metal-to-insulator transition. Instead of the squares used for isotropic systems, one should use rectangles for the anisotropic ones. At the critical point, the ratio of the side lengths must be equal to the squre root of the ratio of the critical values of the quasi-one-dimensional scaling functions. For localized systems, the ratio of the side lengths must be equal to the ratio of the localization lengths.
\end{abstract}

\footnotetext{
${ }^{1}$ (C)2001 The American Physical Society
} 
The presence of disorder[1] may allow a system to make a transition from metallic to insulating behavior by varying the Fermi energy in an energy range where both extended and localized states are found, separated by a mobility edge. Characterizing this transition, one can employ transport properties, such as the conductance, or properties of the system's eigenstates, such as the correlation length for extended, metallic states or the localization length $\xi$ for insulating states. At the mobility edge, a determination of the complete probability distribution $p(g)$ of the conductance $g$ (in units of $e^{2} / h$ ) is needed. The critical point of the transition from metallic states to Anderson localized ones[2] is of particular interest. The distributions are well known to be normal and log-normal off the mobility edge towards the extended and the localized regime, respectively, whereas the exact form of the critical distribution is still under investigation. $[3,4,5,6,7,8,9,10]$ For example, contrary to expectations, the critical distribution seems to vary even within the same universality class, depending on the boundary conditions perpendicular to the direction in which transport occurs. [3, 4, 5] Also, questions about the exact form of the large- $g$ tail $(g>1)$ remain unanswered. Where calculations in $2+\varepsilon$ dimensions[6] indicate higher cumulants to diverge with system size, leading to a power law tail, numerical calculations[7] in three dimensions and analytical results for quasi-one-dimensional wires[5] show an exponential decay.

Anisotropic systems have recently been the focus of particular attention.[11, 12, 13, $14,15,16]$ It is generally accepted that anisotropy does not change the universality class and that isotropic results can be recovered by performing a proper scaling of the anisotropic results. For anisotropic systems in a localized state, it is reasonable to assume that scaling the dimensions of the system by the corresponding localization lengths will make the system effectively isotropic. This procedure has been applied successfully[11] to the scaling function $\Lambda=\lambda_{M} / M$, which is a function of $\xi / M$, where $\lambda_{M}$ denotes 
the finite size localization length of a quasi-one-dimensional strip of finite width $M$ and $\lambda_{M} \rightarrow \xi$ as $M \rightarrow \infty$. It was also shown[17] that the same scaling procedure works for the probability distribution in such a system.

In order to test the approach for critical states one must either face the numerical challenge of large three-dimensional systems or take into account additional interactions (beyond the disorder potential) such as spin-orbit coupling.[18] Another possibility is the introduction of external magnetic fields as, e.g., in integer quantum Hall systems[19] or tight-binding models with random magnetic flux. [20] However, as a result of Anderson localization, extended states do not exist in two-dimensional systems of noninteracting electrons in a magnetic field, except at a singular energy near the center of each of the Landau subbands. At these critical energies $E_{c}$ the localization length $\xi$ diverges with a critical exponent $\nu: \xi \propto\left|E-E_{c}\right|^{-\nu}$.

Because significant finite size effects have to be expected, we decided to concentrate our research on two-dimensional systems, although the exact form of the critical distribution of the conductance depends on the dimensionality of the system.[7] The investigation of the self-averaging quantity $\xi$ in integer quantum Hall systems yielded very encouraging results,[15] supporting the expectation that quantities of anisotropic systems can indeed be mapped to isotropic values by a simple rescaling scheme.

In this paper, we show a method of mapping the probability distributions of the conductance of anisotropic two-dimensional systems with a magnetic field perpendicular to the plane or with spin-orbit coupling to the probability distribution of the conductance for the corresponding isotropic system at the critical point, using a tight-binding model. It turns out that the ratio of the squares of the side lengths $L_{x}, L_{y}$ of the anisotropic system should be chosen equal to the ratio of the critical values $\Lambda_{x}^{c}, \Lambda_{y}^{c}$ of the quasi-onedimensional scaling functions: 


$$
\frac{L_{x}^{2}}{L_{y}^{2}}=\frac{\Lambda_{x}^{c}}{\Lambda_{y}^{c}} .
$$

In the following, we first describe the models and the numerical method we employed. Then we present and discuss our numerical results and finally summarize the conclusions of this work.

The tight-binding model uses the Hamiltonian

$$
\mathcal{H}=\sum_{n, \tau}|n \tau\rangle \varepsilon_{n}\left\langle n \tau\left|+\sum_{n, \tau, n^{\prime}, \tau^{\prime}}\right| n \tau\right\rangle V_{n, n^{\prime}}\left\langle n^{\prime} \tau^{\prime}\right|,
$$

where $n, n^{\prime}$ denotes the lattice site. Without spin-orbit interaction the "variables" $\tau, \tau^{\prime}$ take on only one value and the hopping integrals $V_{n, n^{\prime}}$ are scalar, otherwise they are $2 \times 2$ matrices and the spin variables take on the values 1 or -1 . In either case the site energies $\varepsilon_{n}$ are independent of $\tau$ and we take into account interactions only between neighboring lattice sites.

An external magnetic field enters the Hamiltonian via its vector potential $\mathbf{A}(\nabla \times \mathbf{A}=$ $\mathbf{B})$, which appears in the phases of the hopping integrals:

$$
V_{n, n^{\prime}}=t_{n, n^{\prime}}^{0} \exp \left(-2 \pi i(e / h) \int_{\mathbf{r}_{n}}^{\mathbf{r}_{\mathbf{n}^{\prime}}} \mathbf{A}(\mathbf{r}) \mathrm{d} \mathbf{r}\right)
$$

The integral connects the lattice sites $n$ (at $\mathbf{r}_{n}$ ) and $n^{\prime}\left(\right.$ at $\mathbf{r}_{n^{\prime}}$ ) in a straight line. For the sytems under consideration, where the magnetic induction $\mathbf{B}$ is perpendicular to the plane of the two-dimensional lattice, the gauge for the vector potential can be chosen such that the phases vanish in the direction perpendicular to $\mathbf{A}$ and are integer multiples of some number $2 \pi \alpha$ in the direction parallel to $\mathbf{A}$. The value of the parameter $\alpha$ then completely characterizes the influences of the magnetic field on the system. For rational $\alpha$, the denominator determines the number of bands in the density of states of the system without disorder. 
The Evangelou-Ziman model[21] incorporates spin-orbit coupling by using the following hopping integrals:

$$
V_{n, n^{\prime}}^{\tau, \tau^{\prime}}=t_{n, n^{\prime}}^{0}\left[\delta_{\tau, \tau^{\prime}}+\mu i \sum_{\nu} \sigma_{\tau, \tau^{\prime}}^{\nu} t_{n, n^{\prime}}^{\nu}\right],
$$

where $\nu=x, y, z$ and $\sigma^{\nu}$ are the Pauli matrices. The parameter $\mu$ characterizes the strength of the spin-orbit interaction.

Both systems may be made anisotropic by chosing the value of $t^{0}$ to be different in the two directions within the plane. Otherwise this parameter is a constant, independent of lattice site $n$. We bring disorder to the system by chosing all the site energies independently from a rectangular distribution of width $W$ centered at 0 , so that $W$ is a measure of the strength of the disorder. The parameters $t^{\nu}$ are also randomly selected from a uniform distribution on $[-1 / 2,1 / 2]$. The energy scale is set by the larger of the two values for $t^{0}$, which is taken to be unity.

We calculate the conductance from the Landauer formula[22]

$$
g=\operatorname{Tr}\left(t^{\dagger} t\right)
$$

where $t$ is the transmission matrix. We suppose two semi-infinte leads are attached to opposite sides of the sample. Then $t$ determines the transmission of an electron through the sample. The numerical procedure is based on the algorithms published by Ando[23] and by Pendry et al. [23]

The critical conductance distributions we calculated for isotropic quantum Hall systems at different disorder strengths show that finite-size effects become stronger the weaker the disorder. Where systems with $W=4.0$ and $W=2.0$ show a basically sizeindependent critical distribution of the conductance for squares of $64 \times 64$ lattice sites, at $W=0.5$ finite-size effects are still somewhat noticeable up to systems with $192 \times 192$ lattice sites. The anisotropic quantum Hall systems we investigated are characterized 
by $\alpha=\frac{1}{8}$. The anisotropies we chose were $t_{x}^{0} / t_{y}^{0}=0.5$ at $W=0.5$ and $t_{x}^{0} / t_{y}^{0}=0.8$ at $W=0.1$. The latter was chosen mainly because we already had the data for the quasione-dimensional scaling function. As the disorder is even weaker than in the first case, finite-size effects are even stronger, and even at $240 \times 240$ lattice sites the conductance distribution is far from the one we expect from our calculations of isotropic systems. Therefore, we will not be able to show that our procedure maps the two anisotropic conductance distributions to the critical distribution of isotropic systems for this extreme case. We will, however, be able to prove the somewhat weaker claim that our method transforms the two anisotropic distributions so that both have the same shape. In a square system, one expects that the distribution in the difficult hopping direction shows a more localized character than the one in the easy hopping direction. In an isotropic system, the distribution obviously cannot depend on the direction of transport. By making the system rectangular rather than square, i.e., shorter in the difficult hopping direction, it should be possible to obtain distributions in the two directions that are the same, thus making the anisotropic system effectively behave isotropically.

The task now is "How do we choose the correct ratio of side lengths of the rectangle?" From the research on localized systems $[11,17]$ we know that in those cases, the ratio should be equal to the ratio of the localization lengths:

$$
\left(\frac{L_{x}}{\xi_{x}}=\frac{L_{y}}{\xi_{y}} \quad \Longrightarrow\right) \quad \frac{L_{x}}{L_{y}}=\frac{\xi_{x}}{\xi_{y}}
$$

for localized systems, as these are obviously the appropriate length scales in their respective directions. This is of no use for critical systems as both localization lengths diverge at the transition. A closely corresponding nondiverging quantity is, however, available in the scaling function $\Lambda_{M}=\lambda_{M} / M$, which has a finite critical value, independent of the system width $M$. The finite-size localization lengths $\lambda_{M, x}$ and $\lambda_{M, y}$ have $\xi_{x}$ and $\xi_{y}$, respectively, as their large- $M$ limits, and for large enough systems we can approximate 
Eq. (3.6) by

$$
\frac{L_{z}}{I_{y}}=\frac{\lambda_{M, x}}{\lambda_{M, y}}
$$

for localized systems at "large enough" $M$. The meaning of $M$ in this context would be that of the system width perpendicular to the direction in which the localization length is measured, i.e., $M=L_{y}$ in $\lambda_{M, x}$ and $M=L_{x}$ in $\lambda_{M, y}$. Now, by multiplying both sides of Eq. (3.7) by $L_{x} / L_{y}$ we have

$$
\frac{L_{x}^{2}}{L_{y}^{2}}=\frac{L_{x}}{\lambda_{M, y}} \frac{\lambda_{M, x}}{L_{y}}=\frac{\Lambda_{M, x}}{\Lambda_{M, y}}
$$

for large localized systems. Now $\Lambda_{M}$ is a continuous function of $E$ and for large enough systems at $E_{c}$ should have reached its critical value. Therefore, we arrive at the conclusion that

$$
\frac{L_{x}}{L_{y}}=\sqrt{\frac{\Lambda_{x}^{c}}{\Lambda_{y}^{c}}}
$$

should be the correct ratio for critical systems in order to make them behave like isotropic ones. Noting the relationship $[9,13,17]$

$$
\sqrt{\Lambda_{x}^{c} \Lambda_{y}^{c}}=\Lambda_{\text {iso }}^{c}
$$

we can write Eq. (3.9) in the alternate form

$$
\frac{L_{x}}{L_{y}}=\frac{\Lambda_{x}^{c}}{\Lambda_{\text {iso }}^{c}}=\frac{\Lambda_{\text {iso }}^{c}}{\Lambda_{y}^{c}} .
$$

We tested this prediction on the system with $t_{x}^{0} / t_{y}^{0}=0.5$ and $W=0.5$, where the ratio $\sqrt{\Lambda_{x}^{c} / \Lambda_{y}^{c}}$ is roughly 1.5. The result is shown in Fig. 3.1 together with the critical distribution for the isotropic system. The agreement is very good. For the other system with $t_{x}^{0} / t_{y}^{0}=0.8$ and $W=0.8$, we have to deal with stronger finite-size effects 


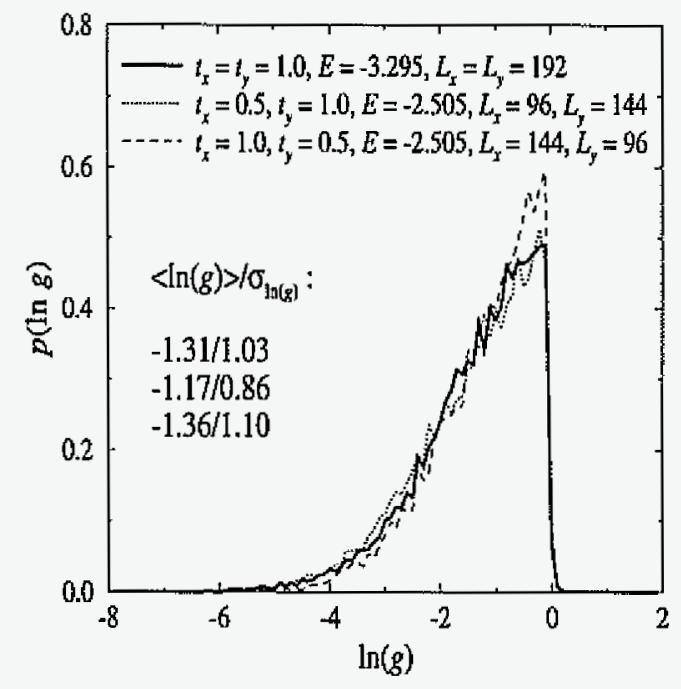

Figure 3.1 The conductance distributions of an anisotropic rectangular system with a ratio of side lengths chosen according to Eq. (3.9). For comparison the corresponding distribution of an isotropic system is shown as well.

and cannot expect to approach the form of the critical distribution we see in Fig. 3.1 for reasonable system sizes. Instead we merely show in Fig. 3.2 how the critical distributions change with the ratio of side lengths. The best value for the ratio according to Eq. (3.9) would be roughly 1.23. Figure 3.2 shows results for ratios of $1.0,1.25$, and 1.5. The averages of $\ln (g)$ for the easy hopping direction decrease with increasing ratio from -4.09 for the square to -4.40 and -5.30 , while the averages for the difficult hopping direction increase from -4.94 for the square to -4.18 and -4.03 . Similarly, the standard deviations increase for the easy hopping direction from 1.90 for the square to 2.07 and 2.30, while they decrease for the difficult hopping direction from 2.24 for the square to 2.01 and 1.91. The values for a ratio of 1.25 are not equal but reasonably close, so that for larger systems, where a ratio of 1.23 might be practicable, we expect a better agreement of the two probability distributions. 


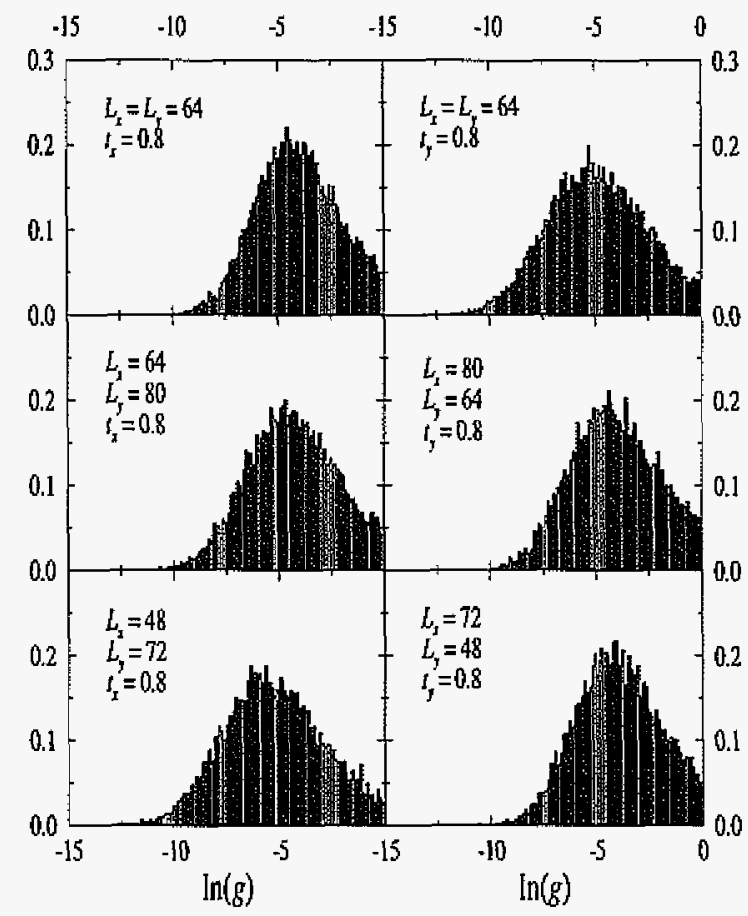

Figure 3.2 Conductance distributions of an anisotropic system for varying ratios of the side lengths. The left panels refer to transport in the easy hopping direction, the right panels to transport in the difficult hopping direction. The ratio of side lenghts is 1.0 for the top row, 1.25 for the middle row, and 1.5 for the bottom row.

Taking the best-ratio rectangle as the "undeformed" base, we can also see from Fig. 3.2 that similar "deformations" have similar effects in the two directions, that is, reducing the ratio by a factor $\gamma$ in one direction will cause the ensemble average $\langle\ln (g)\rangle$ in that direction to increase, and the standard deviation to decrease, while the trend is opposite in the perpendicular direction. However, reducing the ratio by the same factor $\gamma$ in the other direction will result roughly in the same distributions as before, but with the one associated with the easy direction before now assigned to the difficult direction and vice versa.

That the same procedure also works for sytems with spin-orbit coupling is shown in Fig. 3.3, where we plot the conductance distribution for an isotropic system with 
$\mu=1.0$ at $E=0.1$ and $W_{c}=6.7$ together with that of two anisotropic systems, one with $\mu=1.0$ and $t_{x}^{0} / t_{y}^{0}=0.1$ at $E=0.1$ and $W_{c}=1.6$, the other with $\mu=1.0$ and $t_{x}^{0} / t_{y}^{0}=0.2$ at $E=0.1$ and $W_{c}=2.6$. The ratio of sidelengths, according to Eq. (3.9), should be 23.0 for the latter. We chose $40 \times 40$ lattice sites for the isotropic system, $10 \times 230$ lattice sites for the strongly anisotropic one, and $20 \times 185$ lattice sites for the anisotropic system with the weaker anisotropy. Again the agreement is very good.

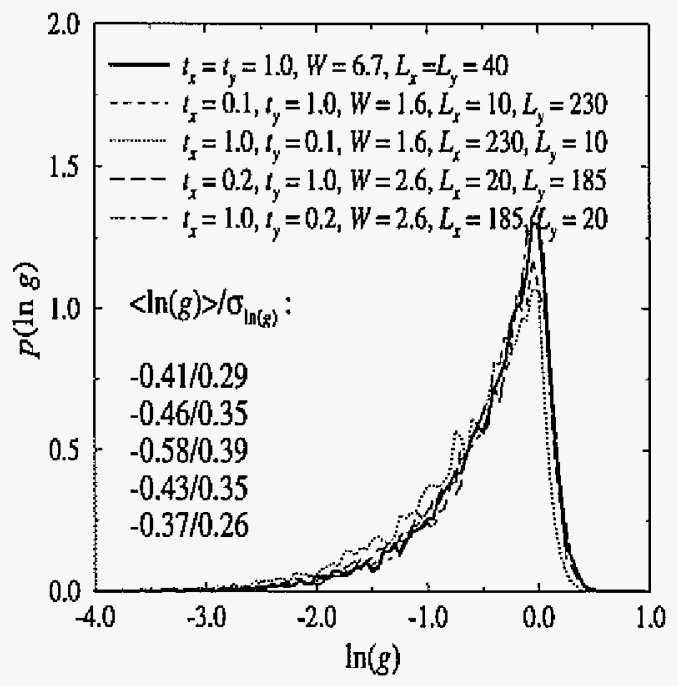

Figure 3.3 The conductance distributions for an isotropic and two anisotropic systems with spin-orbit coupling.

We have shown that the scaling procedure applicable to mapping the conductance distributions of localized anisotropic systems to the corresponding isotropic one can be extended in a straightforward manner to systems at the critical point of the Anderson localization-delocalization transition in both unitary and symplectic two-dimensional systems. Instead of the squares used for isotropic systems, one should use rectangles for the anisotropic ones, with a ratio of side lengths equal to the square root of the ratio of the critical values of the quasi-one-dimensional scaling function. 
Ames Laboratory is operated for the U.S. Department of Energy by Iowa State University under Contract No. W-7405-Eng-82. This work was supported by the Director for Energy Research, Office of Basic Science. P.M. would like to thank IITAP and Ames Laboratory at Iowa State University for their hospitality and support and the Slovak Grant Agency for financial support.

\section{Bibliography}

[1] C. M. Soukoulis and E. N. Economou, Waves Random Media 9, 255 (1999) and references therein.

[2] For a recent review, see B. Kramer, and A. MacKinnon, Rep. Prog. Phys. 56, 1469 (1993).

[3] K. Slevin and T. Ohtsuki, Phys. Rev. Lett. 78, 4083 (1997); K. Slevin, T. Ohtsuki, and T. Kawarabayashi, Phys. Rev. Lett. 84, 3915 (2000); K. Slevin and T. Ohtsuki, Phys. Rev. B 63, 045108 (2001).

[4] C. M. Soukoulis, X. Wang, Q. Li, and M. M. Sigalas, Phys. Rev. Lett. 82, 668 (1999).

[5] D. Braun, E. Hofstetter, G. Montambaux and A. MacKinnon, cond-mat/0101122 (unpublished).

[6] B. Shapiro, Phys. Rev. Lett. 65, 1510 (1990); A. Cohen, Y. Roth, and B. Shapiro, Phys. Rev. B 38, 12125 (1988); B. Shapiro, Philos. Mag. B 56, 1031 (1987).

[7] P. Markoš, Phys. Rev. Lett. 83, 588 (1999).

[8] K. A. Muttalib and P. Wölfle, Phys. Rev. Lett. 83, 3013 (1999).

[9] X. Wang, Q. Li, and C. M. Soukoulis, Phys. Rev. B 58, 3576 (1998). 